\title{
Study of the causes of non-acceptance of VBAC in patients with previous one LSCS in a tertiary institution
}

\author{
Deepa B. Karmali*, Guruprasad Pednekar, Ajit A. Nagarsenkar
}

Department of Obstetrics and Gynecology, Goa Medical College, Goa, India

Received: 18 September 2018

Accepted: 22 October 2018

\section{*Correspondence:}

Dr. Deepa B. Karmali,

E-mail: karmalideepa@gmail.com

Copyright: (C) the author(s), publisher and licensee Medip Academy. This is an open-access article distributed under the terms of the Creative Commons Attribution Non-Commercial License, which permits unrestricted non-commercial use, distribution, and reproduction in any medium, provided the original work is properly cited.

\begin{abstract}
Background: Many women who are eligible candidates for a VBAC (Vaginal Birth after Caesarean) in the current pregnancy opt for a repeat LSCS (Lower Segment Caesarean Section) as a mode of delivery. We decided to evaluate the proportion of patients who opt out of a VBAC and to study the causality of this trend.

Methods: All patients who presented to the Dept. of OBG in our institution and were eligible to have a VBAC were included in the study till the sample size of 100 cases was met. Consultant opinion was sought prior to deciding the mode of delivery.

Results: Incidence of patients of previous one LSCS who were not willing for VBAC was 4\%, out of total number of deliveries and $30.7 \%$, out of all cases of patients with previous one LSCS. The reason for not opting for a VBAC in the current pregnancy in the majority of the eligible patients was the desire for a concurrent sterilization procedure. Others cited apprehension of a scar rupture or fear of labor pain or both as their primary reason for opting out of a VBAC.

Conclusions: The high incidence of ERC (Elective Repeat Caesarean) as compared to TOLAC (Trial of Labour after Caesarean) has long-term clinical and financial implications. Counseling given to the pregnant woman with an emphasis on the advantages of VBAC in order that she opts for a VBAC with confidence and enthusiasm is the only way forward.
\end{abstract}

Keywords: Caesarean section, Previous LSCS, VBAC refusal

\section{INTRODUCTION}

Caesarean section has become the commonest surgery performed on women during the child bearing years. ${ }^{1}$ In the subsequent pregnancy after caesarean section, the mode of delivery is either an elective caesarean section (ERC) or a vaginal birth after caesarean section (VBAC). ${ }^{1}$ Though vaginal delivery after caesarean section (VBAC) is actively encouraged, numbers of women willing to attempt VBAC are on the decline. ${ }^{2}$ It was also seen that when rates of VBAC increased there was also a rise in the associated complications like uterine rupture. ${ }^{3}$ This resulted in worsening the perception towards VBAC. The routine use of transverse incision on the lower segment of the uterus during caesarean section gives a good healing and a subsequent strong scar on the uterus so that the women can withstand labour easily in the next pregnancy, however, a sense of security makes the mother and physician opt for a repeat caesarean section. ${ }^{4,5}$ Patients with previous two or more cesareans are always offered an elective repeat cesarean section (ERCS) in present institution whereas patients with previous one LSCS are offered a VBAC (Vaginal Birth after Caesarean) provided they satisfy the prerequisites for a VBAC. Majority of women who had an LSCS in the previous pregnancy for a multitude of 
non-recurring indications are eligible for a VBAC; however, they do not consent for a vaginal delivery in this pregnancy. We decided to study this trend to observe the proportion of patients who opt out of having a VBAC and to evaluate the causes for the same.

The objectives of the present study were to study the proportion of candidates those are eligible for; but are not willing for VBAC. To study the reasons for nonacceptance of VBAC.

\section{METHODS}

This study was conducted in the Department of OBG in a tertiary institute. Patients with previous one LSCS who are eligible for a VBAC in the current pregnancy. The design of the study was observational and descriptive in nature.

\section{Inclusion criteria}

- Women with a single prior caesarean section, singleton pregnancy in cephalic presentation at $\geq 37$ weeks with no cephalo-pelvic disproportion as decided by the treating consultant.

- Absence of complicating obstetric factor precluding a vaginal delivery in this pregnancy like placenta praevia, transverse lie, multiple pregnancy etc.

\section{Exclusion criteria}

- Women with more than one prior caesarean section; previous vertical/ inverted $\mathrm{T}$ incision.

- Women with previous uterine rupture or perforation, previous uterine surgeries like hysterotomy or myomectomy which entail opening of uterine cavity.
All patients who presented to the Dept. of OBG and who were eligible for but not willing for a VBAC were included in the study till the sample size of 100 cases was met.

Consultant opinion was taken in deciding the mode of delivery. Interview was conducted with the help of a questionnaire. Subjects were explained about their eligibility for a VBAC and the risks associated with a VBAC. Informed consent was taken.

\section{RESULTS}

We interviewed pregnant patients with previous one LSCS who reported to the department of OBG over a period of six months from October 2015 to March 2016 till the sample size of 100 cases was met. The total number of deliveries during this period was 2478 out of which 1663 were vaginal deliveries $(67.1 \%)$ while 815 were LSCS deliveries (32.8\%).

The total number of patients with previous one LSCS was $325(13.1 \%)$. Of these, 100 were not willing for VBAC and hence were included in the study. A total of 165 patients were taken for LSCS for other indications. We had a total of $60 \mathrm{VBAC}$ deliveries in this study period giving a proportion of $18.5 \%$. The results obtained were as follows:

- Age: The majority of the patients were spread over the age group of 20-35 years with the maximum number in the 20-25 age groups.

- Education: Most of the patients were educated up to twelfth standard.

- Religion: Maximum numbers of the patients were hindus (Table 1).

Table 1: Socio-demographic variables.

\begin{tabular}{|c|c|c|c|c|c|c|c|}
\hline \multirow{2}{*}{ Age } & $\leq 20$ & $21-25$ & $26-30$ & 31-35 & $36-40$ & $\geq 40$ & Total \\
\hline & 1 & 35 & 23 & 28 & 12 & 1 & 100 \\
\hline \multirow{2}{*}{ Education } & $\leq \mathrm{X}$ & & X-XII & & Graduate & & \multirow{2}{*}{100} \\
\hline & 32 & & 57 & & 11 & & \\
\hline \multirow{2}{*}{ Religion } & Hindu & & Muslim & & Christian & & \multirow{2}{*}{100} \\
\hline & 76 & & 17 & & 7 & & \\
\hline
\end{tabular}

- Gravida: The highest numbers of patients were in their second pregnancy (Table 2).

Table 2: Obstetric profile of the participants.

\begin{tabular}{|lllll|}
\hline Gravida & Second & Third & Fourth & Total \\
& $\mathbf{6 8}$ & $\mathbf{2 3}$ & $\mathbf{9}$ & $\mathbf{1 0 0}$ \\
\hline Gestation & $\leq 37$ & $37.1-40$ & $\geq 40.1$ & Total \\
\cline { 2 - 5 } in weeks & 17 & 82 & 1 & 100 \\
\hline
\end{tabular}

- Period of gestation: Most were at term, that is, between 37 and 40 weeks.

- Indication for previous LSCS: The indications for the previous LSCS done in these patients were varied. The maximum were done for meconium stained amniotic fluid followed by CPD (Figure 1).

Incidence of patients of previous one LSCS who were not willing for VBAC out of total number of deliveries was 
found to be $4 \%$ whereas it was found to be $30.7 \%$ of all cases of patients with previous one LSCS.

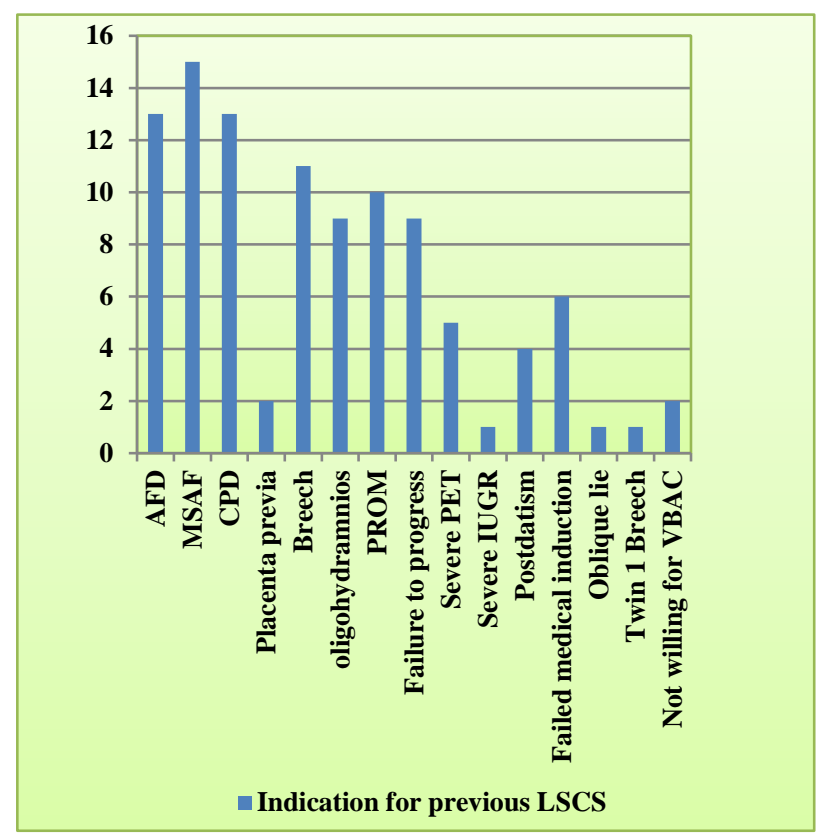

Figure 1: Indications for previous LSCS.

- Reason for not wanting a VBAC in the current pregnancy: Majority of the patients opted out of having a VBAC in the current pregnancy despite being eligible to undergo it as they wanted to have a concurrent sterilization procedure. Others cited fear of a scar rupture or fear of labor pain or both as their primary reason for opting out of a VBAC (Table 3).

Table 3: Reasons for refusal of VBAC.

\begin{tabular}{|l|l|}
\hline Reasons & No. \\
\hline Desirous of concurrent ligation & 55 \\
\hline Fear of scar rupture & 14 \\
\hline Fear of pain & 27 \\
\hline Fear of pain and scar rupture & 4 \\
\hline Total & 100 \\
\hline
\end{tabular}

\section{DISCUSSION}

The incidence of LSCS both primary and repeat is continuously on the rise. ${ }^{6}$ This rising incidence of LSCS worldwide is a big concern today in obstetrics. ${ }^{7}$ This is mainly due to cases of breech presentation and of previous LSCS needing a repeat LSCS. ${ }^{8}$ The number of women opting for VBAC have decreased and importantly it has been shown to be due to a change in the doctor and patient perception of the dangers involved in a VBAC rather than a change in the characteristics in the clinical profile of the patient. ${ }^{9}$

More than half of the women in the study group (55\%) opted for an ERC as they wanted a concurrent sterilization at the time of the second LSCS. This was in spite of the explanation that a small mini laparotomy incision for a puerperal ligation is always better than a big incision for a major laparotomy and the complications in the former are less as well. The remaining women cited fear of labor pain, fear of scar rupture or both as the reason for refusal of a VBAC. Cases of maternal request for a repeat LSCS are on the rise. ${ }^{5}$ Women refusing VBAC are contributing to the increasing rates of caesarean section. ${ }^{10}$ The preference of the woman always has to be given importance. ${ }^{11}$ Women preference for the mode of delivery is influenced by their personal, social factors and their understanding of the risks and benefits. ${ }^{2}$ It also depends on the attitude of the family, community and health care professionals involved. ${ }^{12}$ It is not advisable to force a women unwilling for VBAC to undergo vaginal delivery. ${ }^{13}$ The woman and her obstetrician together should make an informed choice. ${ }^{13}$

Counseling forms a very important part of the management of such cases. Both modes of delivery namely, VBAC and ERC have their own advantages and disadvantages. ${ }^{1}$ Patients and family should be informed about chances of abnormal placentation like placenta previa and morbidly adherent placenta in pregnancies subsequent to LSCS. $^{14}$ Extensive and sustained counseling throughout the antenatal period and more importantly at term, given to the pregnant woman, with an emphasis on the advantages of VBAC so that she opts for a VBAC with confidence and enthusiasm is the only way forward.

\section{Funding: No funding sources}

Conflict of interest: None declared

Ethical approval: The study was approved by the Institutional Ethics Committee

\section{REFERENCES}

1. Crowther CA, Dodd JM, Hiller JE, Haslam RR, Robinson JS, Group on behalf of the BACS. Planned Vaginal Birth or Elective Repeat Caesarean: Patient Preference Restricted Cohort with Nested Randomised Trial. Smith GC, editor. PLoS Med. 2012;9(3):1-10.

2. Dodd JM, Crowther CA, Hiller JE, Haslam RR, Robinson JS. Birth after caesarean study--planned vaginal birth or planned elective repeat caesarean for women at term with a single previous caesarean birth: protocol for a patient preference study and randomised trial. BMC Pregnancy Childbirth. 2007;7(1):17.

3. Birth V, Previous A. ACOG Practice bulletin no. 115: Vaginal birth after previous cesarean delivery. Obstet Gynecol. 2010;116(2 Pt 1):450-63.

4. Goel S, Tiwari M, Hariharan C, Shrivastava D. Outcome of post caesarean pregnancy and comparison of maternal and foetal outcome following vaginal birth versus repeat caesarean section in a rural hospital. Int $\mathbf{J}$ Reprod Contraception, Obstet Gynecol. 2013;2(1):16-22. 
5. Jawa A, Garg S, Tater A SU. Indications and rates of lower segment caesarean section at tertiary care hospital-an analytical study. Int J Reprod Contracept Obs Gynecol. 2016;5(10):3466-9.

6. Badge VL, Aggarwal SS, Ambalkar DD, Humne A, Raghuwanshi N. Assessment of indications of lower section caesarean section at tertiary care centre: a cross sectional study. Int $\mathrm{J}$ Community Med Public Heal. 2017;4(4):1253-6.

7. Bala S, Patidar BL, Gupta B. A Retrospective Analysis of Annual Cesarean Section Rate in a Tertiary Care Hospital, KOTA. J Med Sci Clin Res. 2017;05(07):25325-9.

8. Bragg F, Cromwell DA, Edozien LC, Gurol-Urganci I, Mahmood TA, Templeton A, et al. Variation in rates of caesarean section among English NHS trusts after accounting for maternal and clinical risk: Cross sectional study. BMJ. 2010;341(c5065):1-8.

9. Grobman WA, Lai Y, Landon MB, Spong CY, Rouse DJ, Varner MW, et al. The change in the rate of vaginal birth after caesarean section. Paediatr Perinat Epidemiol. 2011;25(1):37-43.

10. Birth After Previous Caesarean. Green-top Guideline No. 45. 2007;(45):1-17.
11. Schoorel E, Vankan E, Scheepers H, Augustijn B, Dirksen CD, De Koning M, et al. Involving women in personalised decision-making on mode of delivery after caesarean section: the development and pilot testing of a patient decision aid. BJOG. 2014;121(2):202-9.

12. Rozen G, Ugoni AM, Sheehan PM. A new perspective on VBAC: A retrospective cohort study. Women and Birth. 2011;24(1):3-9.

13. Scott J. Intrapartum management of trial of labour after caesarean delivery: evidence and experience. BJOG. 2014;121(2):157-62.

14. Nikhil A, Desai A, Vijay K, Seema P, Bhumika K, Riddhi P. Analysis of trends in lscs rate and indications of lscs: a study in a medical colleege hospital gmers, Sola, Ahmedabad. Int J Pharm bio Sci. 2015;2(1):1-5.

Cite this article as: Karmali DB, Pednekar G, Nagarsenkar AA. Study of the causes of nonacceptance of VBAC in patients with previous one LSCS in a tertiary institution. Int J Reprod Contracept Obstet Gynecol 2018;7:4945-8. 